\title{
Separate Transport Systems for Biliary Secretion of Sulfated and Unsulfated Bile Acids in the Rat
}

\author{
Folkert Kuipers, Martin Enserink, Rick Havinga, Ad B. M. van der Steen, Machiel J. Hardonk, Johan Fevery, and Roel J. Vonk \\ Department of Pediatrics, Department of Pathology, and Central Animal Laboratory, University of Groningen, Groningen, \\ The Netherlands; and Department of Internal Medicine, University of Leuven, Leuven, Belgium
}

\begin{abstract}
Biliary secretion of $3 \alpha$-sulfated bile acids has been studied in Wistar rats with an autosomal recessive defect in the hepatic transport of bilirubin. Liver function, established by measurement of various enzymes in plasma, by enzyme histochemical methods, and by electron microscopy, appeared to be normal in these rats. Serum levels of unconjugated, monoglucuronidated, and diglucuronidated bilirubin were $0.62,1.62$, and $6.16 \mu \mathrm{mol} /$ liter, respectively, compared with $0.17,0.08$, and $0.02 \mu \mathrm{mol} /$ liter in control rats. Biliary bilirubin secretion was strongly reduced in the mutant animals: $0.21 \pm 0.03$ vs. $0.39 \pm 0.03$ $\mathrm{nmol} / \mathrm{min}$ per $100 \mathrm{~g}$ body wt in control rats. Despite normal biliary bile acid output, bile flow was markedly impaired in the mutant animals, due to a 53\% reduction of the bile acid-independent fraction of bile flow. The transport maximum for biliary secretion of dibromosulphthalein (DBSP) was also drastically reduced $(-53 \%)$.

Biliary secretion of intravenously administered trace amounts of the $3 \alpha$-sulfate esters of ${ }^{14} \mathrm{C}$-labeled taurocholic acid $(-14 \%)$, taurochenodeoxycholic acid $(-39 \%)$, taurolithocholic acid $(-73 \%)$, and glycolithocholic acid $(-91 \%)$ was impaired in the jaundiced rats compared with controls, in contrast to the biliary secretion of the unsulfated parent compounds. Hepatic uptake of sulfated glycolithocholic acid was not affected in the jaundiced animals. Preadministration of DBSP (15 $\mu \mathrm{mol} / 100$ $\mathrm{g}$ body wt) to normal Wistar rats significantly impaired the biliary secretion of sulfated glycolithocholic acid, but did not affect taurocholic acid secretion.

We conclude that separate transport systems in the rat liver exist for biliary secretion of sulfated and unsulfated bile acids; the sulfates probably share secretory pathways with the organic anions bilirubin and DBSP. The described genetic defect in hepatic transport function is associated with a reduced capacity to secrete sulfated bile acids into bile; this becomes more pronounced with a decreasing number of hydroxyl groups on the sulfated bile acid's molecule.
\end{abstract}

\section{Introduction}

Sulfation has been recognized as an important pathway in bile acid metabolism since the first demonstration of sulfate esters

Address reprint requests to Dr. Kuipers, Dept. of Pediatrics, Bloemsingel 10, $9712 \mathrm{KZ}$ Groningen, The Netherlands.

Received for publication 7 April 1987 and in revised form 4 December 1987.

J. Clin. Invest.

(C) The American Society for Clinical Investigation, Inc.

$0021-9738 / 88 / 05 / 1593 / 07 \quad \$ 2.00$

Volume 81, May 1988, 1593-1599 of lithocholic acid in human bile in 1967 (1). Considerable amounts of sulfated bile acids are found in the urine of man under cholestatic conditions $(2,3)$. However, it has been well established that in absence of cholestasis, the biliary pathway is quantitatively the main route for secretion of sulfated bile acids, both in man (4-6) and in experimental animals $(7,8)$. The mechanisms of hepatic bile acid transport, i.e., uptake from the bloodstream by hepatocytes, intracellular transport, and excretion at the canalicular pole of the cells, are still poorly defined. It is generally assumed that carrier-mediated transport across the sinusoidal as well as the canalicular membranes is involved $(9,10)$. Whether sulfated bile acids are secreted by the same carrier systems as the unsulfated compounds is not known.

In the present study we have compared the biliary secretion of sulfated bile acids in normal rats and in rats with a genetic defect in the biliary secretion of bilirubin. The results of this study indicate that sulfated bile acids are secreted into bile by a system distinct from that for unsulfated bile acids.

\section{Methods}

Materials. ${ }^{14} \mathrm{C}$-Taurocholic acid (TC; ${ }^{1}$ specific activity, $56 \mathrm{mCi} / \mathrm{mmol}$ ) and ${ }^{3} \mathrm{H}-\mathrm{TC}(2.7 \mathrm{Ci} / \mathrm{mmol})$ were obtained from Amersham International (Amersham, UK) and New England Nuclear (Boston, MA), respectively. ${ }^{14} \mathrm{C}$-Taurolithocholic acid (TLC, $3.0 \mathrm{mCi} / \mathrm{mmol}$ ), ${ }^{14} \mathrm{C}-$ glycolithocholic acid (GLC, $6.5 \mathrm{mCi} / \mathrm{mmol}$ ), and ${ }^{14} \mathrm{C}$-taurochenodeoxycholic acid (TCDC, $2.31 \mathrm{mCi} / \mathrm{mmol}$ ) were purchased from CBN, La Jolla, CA. Unlabeled bile acids, all A grade, came from Calbiochem-Behring Corp., La Jolla, CA. Sulfate esters of labeled bile acids were synthetized using a sulfur trioxide pyridine complex (Janssen Pharmaceutica B.V., Beerse, Belgium) as a sulfating reagent. Sulfated bile acids were separated from unreacted material by anion exchange chromatography on Lipidex-DEAP (Packard, Groningen, The Netherlands), and subsequently desalted on a Sep-Pak $C_{18}$ cartridge (Waters Assoc., Div. of Millipore Corp., Milford, MA). Small amounts of disulfated TC were separated from its monosulfates as described by Eyssen et al. (11). Purity of the $3 \alpha$-sulfates was checked by thin-layer chromatography (12) followed by zonal scanning of the plates. Dibromosulphthalein (DBSP) was obtained from SERB, Paris, France.

Animals. Wistar rats, originally obtained from TNO, Zeist, The Netherlands, were bred at the Central Animal Laboratory, University of Groningen. A litter was observed in which three out of eleven siblings showed symptoms of jaundice. Subsequent breeding experiments revealed a pattern of inheritance consistent with an autosomal recessive trait. Homozygous rats were obtained which showed normal feeding patterns and weight gain, and also reproduced normally. Up to now, four generations have been bred. Only male rats have been used for experiments; normal male Wistar rats were taken as controls.

1. Abbreviations used in this paper: BAIF, bile acid-independent fraction; DBSP, dibromosulphthalein; GLC, glycolithocholic acid; STC, STCDC, STLC, and SGLC, sulfated TC, TCDC, TLC, and GLC, respectively; TC, taurocholic acid; TCDC, taurochenodeoxycholic acid; TLC, taurolithocholic acid. 
Liver morphology. Histological and histochemical investigations were performed in five jaundiced and five control rats. Livers were excised and a piece of the large liver lobe was frozen in liquid freon $\left(-90^{\circ} \mathrm{C}\right)$. Cryostat sections $(10 \mu \mathrm{m})$ were fixed in $4 \%$ formaldehyde, $5.4 \%$ macrodex, $1 \% \mathrm{CaCl}_{2}$ in $0.9 \% \mathrm{NaCl}$ for $10 \mathrm{~min}$ at $4^{\circ} \mathrm{C}$ and stained with hematoxylin and eosin, or used for enzyme histochemistry. The following enzyme reactions were performed as described (13): 5 '-nucleotidase, adenosine triphosphatase, alkaline phosphatase, and acid phosphatase. Fixation and incubation for the demonstration of leucyl$\beta$-naphtylamidase, $\beta$-glucuronidase, and NADPH-tetrazolium reductase were performed as described by Hardonk et al. (14). Blood was collected in these experiments before excision of the liver by puncturing of the abdominal aorta. Plasma thus obtained was used for determination of liver enzymes, triglycerides, cholesterol, and bile acids.

For electron microscopic studies, livers of two jaundiced and two control rats were processed as described by Hardonk et al. (15).

Experimental procedures. Bilirubin metabolism was studied in pentobarbital-anesthetized rats $(275-300 \mathrm{~g})$. Bile was collected on ice and in the dark over a period of $20 \mathrm{~min}$, starting $5 \mathrm{~min}$ after creation of a bile fistula. During this period the animal's rectal temperature was maintained at $37.5^{\circ}-38^{\circ} \mathrm{C}$ by means of a heating pad. After $20 \mathrm{~min}$ blood was sampled by heart puncture, transferred to heparinized test tubes, and immediately centrifuged. Plasma and bile samples were stored at $-20^{\circ} \mathrm{C}$ until analysis.

For studies on the process of bile formation, the biliary excretion of DBSP, and of sulfated bile acids, we equipped rats with permanent silastic catheters in bile duct, duodenum, and heart, as described in detail elsewhere (13). This technique allows us to interrupt the enterohepatic circulation and to collect bile from unanesthetized, unrestrained rats. Experiments were performed after at least $8 \mathrm{~d}$ of bile diversion, i.e., after establishment of a new steady state in endogenous bile acid synthesis (13). The heart catheter allows sampling of blood and intracardial administration of compounds without disturbing the animals (16). In a number of experiments, 24-h urine production was collected to determine renal secretion of the administered bile acid. For this purpose, rats were housed in metabolic cages.

The transport maximum for biliary secretion of DBSP was determined as described previously (17), after intravenous administration of $15 \mu \mathrm{mol} \mathrm{DBSP} / 100 \mathrm{~g}$ body wt.

Solutions of radiolabeled bile acids were prepared in saline containing $1 \%$ bovine albumin (Sigma Chemical Co., St. Louis, MO) and passed through a 0.22- $\mu \mathrm{m}$ filter (Schleicher \& Schuell, Den Bosch, The Netherlands) before injection. Bile acids were administered via the heart catheter, and bile collection was started in 15-min fractions for 3 h. When indicated, blood samples of $0.15 \mathrm{ml}$ were taken at $1,2,3,4,5$, $6,8,10,15,20,30,45$, and $60 \mathrm{~min}$ after injection and immediately centrifuged to obtain plasma. All experiments were performed from 11 a.m. to 2 p.m.

Analyses. Plasma levels of alkaline phosphatase, lactate dehydrogenase, aspartate aminotransferase, alanine aminotransferase, triglycerides, and cholesterol were determined as described previously (13). Plasma bile acid concentrations were measured by capillary gas chromatography as described by Wolthers et al. (18), after extraction of the bile acids from plasma by means of Sep-Pak $C_{18}$ cartridges.

Bilirubin, as well as the relative amounts of unconjugated, monoglucuronidated, and diglucuronidated bilirubin, were determined by alkaline methanolysis followed by thin-layer chromatography for bile samples (19) and by HPLC for plasma samples (20). Total biliary bile acid concentration was determined by an enzymatic fluorimetric assay (Sterognost-Flu; Nyegaard \& Co., Oslo, Norway). Cholesterol and phospholipids in bile were measured after lipid extraction (21) according to Gamble et al. (22) and Bötcher et al. (23), respectively.

Radioactivity in bile and plasma was determined in 50- $\mu$ l aliquots in an Isocap scintillation counter. Bile was first decolorized by an equal volume of $\mathrm{H}_{2} \mathrm{O}_{2}$. In a number of representative experiments, radiolabeled bile acids excreted into bile were analyzed by HPLC as described by Kuipers et al. (24).
Calculations and statistics. Biliary output rates were calculated by multiplying bile flow and biliary concentration after correction of the latter for the dead space of the tubing system by the computer program EHCBIN (Dr. H. Aling, Technical University Delft, Delft, The Netherlands). Kinetic parameters were calculated from plasma disappearance curves by the curve-stripping computer program RUGFIT (25) by Dr. A. H. J. Scaf, Laboratory of Pharmacology, University of Groningen. When appropriate, statistical significance was evaluated using Student's $t$ test.

\section{Results}

Liver function. Table I shows the activity of a number of hepatic enzymes in plasma, as well as plasma concentrations of lipids, bile acids, and bilirubin in jaundiced rats and in agematched control Wistar rats. No significant differences were observed between the two groups for enzyme activities and plasma lipid concentrations. Bile acid concentration was significantly elevated in the jaundiced rats. Cholic acid was the main bile acid species in both groups (60-70\%). Plasma bilirubin levels were increased 30 -fold in the jaundiced rats, mainly due to very high concentrations of bilirubin diglucuronide.

Liver morphology was investigated by light and electron microscopy. Neither of these studies revealed abnormalities in livers of jaundiced rats. Furthermore, the activity of seven hepatic enzymes (see Methods) were investigated by enzyme histochemical techniques. No differences between jaundiced and control rats were found. Bilirubin secretion into bile, measured in pentobarbital-anesthetized bile fistula rats, was significantly impaired in the jaundiced rats (Table II), which was associated with significant changes in the relative amounts of the bilirubin conjugates in bile. Furthermore, biliary excretion of DBSP was reduced in jaundiced rats compared with the controls. After an intravenous bolus injection of $15 \mu \mathrm{mol} / 100$ body wt of DBSP, the maximal biliary secretion rate was

Table I. Comparison of Plasma Parameters in Age-Matched Jaundiced and Control Male Wistar Rats

\begin{tabular}{lcc}
\hline & Jaundiced rats & Control rats \\
\hline Alkaline phosphatase (U/liter) & $104 \pm 11$ & $124 \pm 14$ \\
Lactate dehydrogenase (U/liter) & $138 \pm 11$ & $154 \pm 18$ \\
Aspartate aminotransferase (U/liter) & $52 \pm 3$ & $59 \pm 1$ \\
Alanine aminotransferase (U/liter) & $64 \pm 4$ & $61 \pm 2$ \\
Triglycerides (mmol/liter) & $1.86 \pm 0.21$ & $2.10 \pm 0.26$ \\
Cholesterol (mmol/liter) & $1.8 \pm 0.1$ & $2.0 \pm 0.1$ \\
Bile acids ( $\mu$ mol/liter) & $14.8 \pm 1.1^{*}$ & $6.6 \pm 0.9$ \\
Bilirubin ( $\mu$ mol/liter) & $8.40 \pm 1.55^{*}$ & $0.27 \pm 0.06$ \\
UCB (\%) & $7.4 \pm 0.5^{*}$ & $64.5 \pm 5.9$ \\
BMG (\%) & $19.3 \pm 0.9$ & $28.3 \pm 4.5$ \\
BDG (\%) & $73.3 \pm 1.3^{*}$ & $8.8 \pm 5.3$ \\
\hline
\end{tabular}

Blood was obtained from pentobarbital-anesthetized rats by puncturing of the abdominal aorta. The activity of hepatic enzymes and the concentrations of cholesterol, triglycerides, bile acids, and bilirubin, as well as the relative amounts of bilirubin conjugates, were determined in plasma as described in Methods. Values are means $( \pm$ SEM) of five rats in both groups. UCB, Unconjugated bilirubin; BMG, bilirubin monoglucuronide; BDG, bilirubin diglucuronide.

* Significant difference $(P<0.05)$ between jaundiced and control rats. 
Table II. Biliary Bilirubin Secretion in Age-Matched Jaundiced and Control Wistar Rats

\begin{tabular}{lcc}
\hline & Jaundiced rats & Control rats \\
\hline Bilirubin secretion & & \\
(nmol/min per $100 \mathrm{~g}$ body wt) & $0.212 \pm 0.026^{*}$ & $0.391 \pm 0.027$ \\
UCB (\%) & $1.8 \pm 0.5^{*}$ & $3.5 \pm 0.3$ \\
BMG (\%) & $39.1 \pm 1.5^{*}$ & $48.7 \pm 0.9$ \\
BDG (\%) & $59.1 \pm 1.1^{*}$ & $47.8 \pm 1.2$
\end{tabular}

Bile was collected on ice and in the dark from pentobarbital-anesthetized bile fistula rats. Bilirubin conjugates were measured as described in Methods. Values are means ( \pm SEM) of five rats in both groups. UCB, unconjugated bilirubin; BMG, bilirubin monoglucuronide; $\mathrm{BDG}$, bilirubin diglucuronide.

* Significant difference $(P<0.05)$ between jaundiced and control rats.

$49.1 \pm 1.7 \mathrm{nmol} / \mathrm{min}$ per $100 \mathrm{~g}$ body wt vs. $105.4 \pm 3.9 \mathrm{nmol} /$ min per $100 \mathrm{~g}$ body wt in the controls $(P<0.05)$. This value represents the maximal transport capacity for biliary secretion of DBSP in conscious Wistar rats with long-term biliary drainage, as was shown previously (17).

Bile formation. Fig. 1 shows the relation between bile flow and biliary bile acid secretion during $6 \mathrm{~h}$ immediately after the interruption of the enterohepatic circulation in jaundiced and control rats. Despite similar bile acid secretion rates, bile flow was significantly lower in the jaundiced rats, due to a $53 \%$ reduction of the bile acid-independent fraction (BAIF) of bile flow in these animals. Endogenous bile acid pool size of jaundiced rats, determined from the washout curves, was similar to that of the controls, $\sim 60 \mu \mathrm{mol} / 100 \mathrm{~g}$ body $\mathrm{wt}$.

After $8 \mathrm{~d}$ of bile diversion, bile flow was still significantly lower in the jaundiced rats $(1.97 \pm 0.07$ vs. $2.85 \pm 0.04 \mu \mathrm{l} / \mathrm{min}$

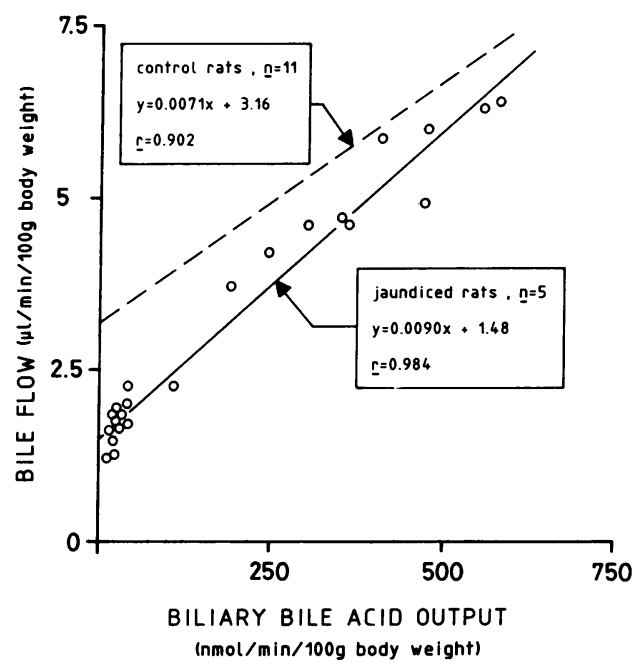

Figure 1. Relation between bile flow and biliary acid excretion in jaundiced and control rats. Jaundiced and control Wistar rats were equipped with permanent catheters in bile duct and duodenum as described (13). Both catheters were immediately connected to each other to maintain an intact enterohepatic circulation. After a recovery period of $8 \mathrm{~d}$, the enterohepatic circulation was interrupted, and bile was sampled for $6 \mathrm{~h}$ in hourly fractions. (Dashed line) Control rats, $n=11$. (Solid line) Jaundiced rats, $n=5$. per $100 \mathrm{~g}$ body wt, $P<0.001)$. In contrast, biliary bile acid secretion, which represents hepatic bile acid synthesis rate under these conditions, and biliary secretion of cholesterol and phospholipids, were similar in both groups due to significantly higher concentrations of these components in bile of the jaundiced rats (bile acids, $29.1 \pm 0.8$ vs. $21.7 \pm 1.6$; cholesterol, $0.87 \pm 0.06$ vs. $0.54 \pm 0.09$; phospholipids, $5.5 \pm 0.2$ vs. $3.7 \pm 0.1$ in jaundiced and control rats, respectively, all values in millimoles/liter).

Biliary secretion of sulfated and unsulfated bile acids. Fig. 2 shows the cummulative biliary secretion of a number of $3 \alpha$ sulfated bile acids and of their unsulfated precursors after intravenous injection of trace amounts in normal Wistar rats and jaundiced animals. The biliary secretion of all sulfated bile acids studied was significantly impaired in the jaundiced rats in comparison with controls. Expressed as percentage of biliary recovery at $1 \mathrm{~h}$ after injection, inhibition was in the order: sulfated TC (STC) (-14.0\%) less than sulfated TCDC (STCDC) $(-39.4 \%)$, less than V sulfated TLC (STLC) $(-73.1 \%)$, less than sulfated GLC (SGLC) (-91.1\%). In contrast, the $1-\mathrm{h}$ biliary recovery of the unsulfated compounds was similar in jaundiced and control animals, exceeding $90 \%$ of the injected amount in almost all cases. The only exception in this respect was GLC, whose biliary secretion was slightly reduced in jaundiced animals $(-14.2 \%, P<0.05)$. All sulfated bile acids were secreted into bile unchanged in form, as revealed by HPLC analysis (data not shown). Unsulfated lithocholic acid conjugates and TCDC were to a variable extent converted to more polar bile acids before their secretion, co-eluting with the muricholic acid fraction in our HPLC system (26) or with their $3 \alpha$-sulfate esters, both in jaundiced and in control rats. TC was not metabolized before its secretion in either of the experiments.

The hepatic handling of TC and of SGLC has been studied in more detail. Fig. 3 shows the biliary secretion rate of simultaneously administered trace amounts of ${ }^{3} \mathrm{H}-\mathrm{TC}$ and ${ }^{14} \mathrm{C}$ SGLC in jaundiced rats, in untreated control rats, and in control rats pretreated with $15 \mu \mathrm{mol} / \mathrm{DBSP}$ per $100 \mathrm{~g}$ body wt, 15 min before administration of the bile acids. From this figure it is evident that the biliary secretion of SGLC was markedly impaired in jaundiced as well as in DBSP-treated control animals, compared with untreated controls, whereas that of TC was not significantly affected in DBSP-treated rats and only slightly delayed in the jaundiced animals. In spite of this, urinary secretion of ${ }^{14} \mathrm{C}$-SGLC was negligible in these experiments $(<2 \%$ dose $/ 24 \mathrm{~h})$.

The plasma disappearance of ${ }^{3} \mathrm{H}$-TC (Fig. 4) was very rapid and almost identical in untreated and DBSP-treated control rats, and in the jaundiced rats; the half-life values were $\sim 0.5$ min in all experiments. Plasma disappearance of ${ }^{14} \mathrm{C}$-SGLC was slower than that of ${ }^{3} \mathrm{H}$-TC in untreated control rats, and strongly delayed in the DBSP-treated controls and in the jaundiced rats. Pharmacokinetic parameters were calculated from the SGLC curves using the fit-program RUGFIT (Table III). The best fit was obtained by the assumption of a two-compartment model with plasma as central compartment and secretion from a second compartment, i.e., the liver. The hepatic uptake of ${ }^{14} \mathrm{C}$-SGLC was not decreased in the jaundiced and DBSP-treated rats, compared with the controls, as appears from similar rate constants for hepatic uptake. The rate constants for biliary secretion were smaller in the former two 


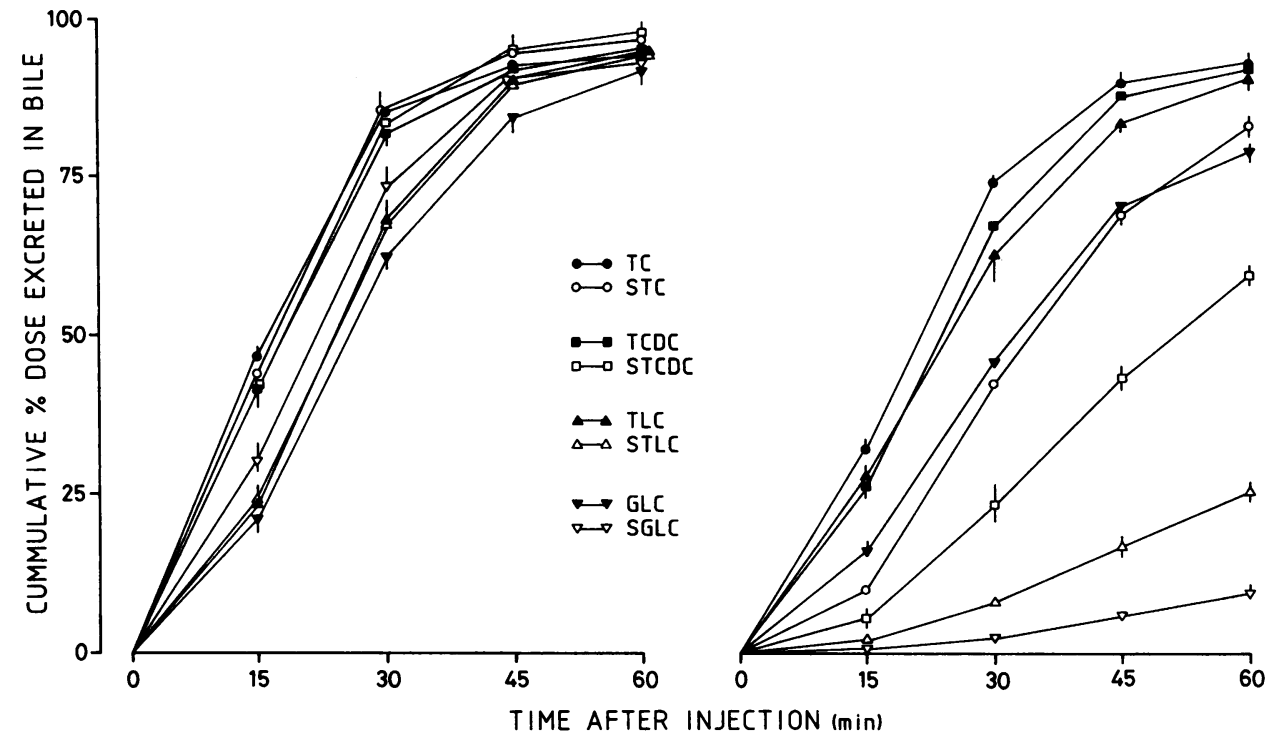

Figure 2. Cummulative biliary secretion of radioactivity after intravenous administration of conjugated bile acids and their $3 \alpha$-sulfate esters to control (left) or jaundiced rats (right). Trace amounts of ${ }^{14} \mathrm{C}$-labeled bile acids were administered to bile-diverted rats via a heart catheter, and bile collection was started immediately in 15-min fractions. Values are means $( \pm$ SEM) of three to five experiments. S, $3 \alpha$-Sulfate ester. groups, albeit not significantly for the DBSP-treated rats. These animals showed a very high rate of reflux from liver to plasma.

\section{Discussion}

In the present study we have compared the hepatic handling of four conjugated, naturally occurring mono-, di-, and trihydroxylated bile acids and their $3 \alpha$-sulfate esters, in a rat strain with an inherited disorder in hepatic bilirubin transport, recently bred in our laboratory. These rats have a markedly reduced capacity to secrete bilirubin conjugates into bile, associated with up to 30-fold increased plasma levels of conjugated bilirubin without morphological or enzyme histochemical signs of cholestasis. Bilirubin in plasma and bile was predominantly in the form of its diglucuronide. This clearly distin- guishes these rats from Gunn rats, in which the activity of the enzyme bilirubin UDP-glucuronyl transferase, which catalyzes the conversion of bilirubin to its glucuronides, is deficient (27). The biochemical defect in our rats has yet to be characterized. It affects the hepatic transport of certain organic anions, comparable with the defect in patients with Dubin-Johnson syndrome (28) and in mutant Corriedale sheep (29). The maximal transport capacity for the organic anion DBSP was reduced by $53 \%$ in the jaundiced animals. On the other hand, the secretion of endogenous unsulfated bile acids, another class of organic anions, was not affected. Despite normal biliary bile acid secretion rate, bile flow was significantly impaired in the jaundiced rats, due to a 53\% reduction of the so-called bile BAIF of bile flow. The observed $46 \%$ reduction in biliary bilirubin secretion suggests that the biliary secretion of this "non-bile

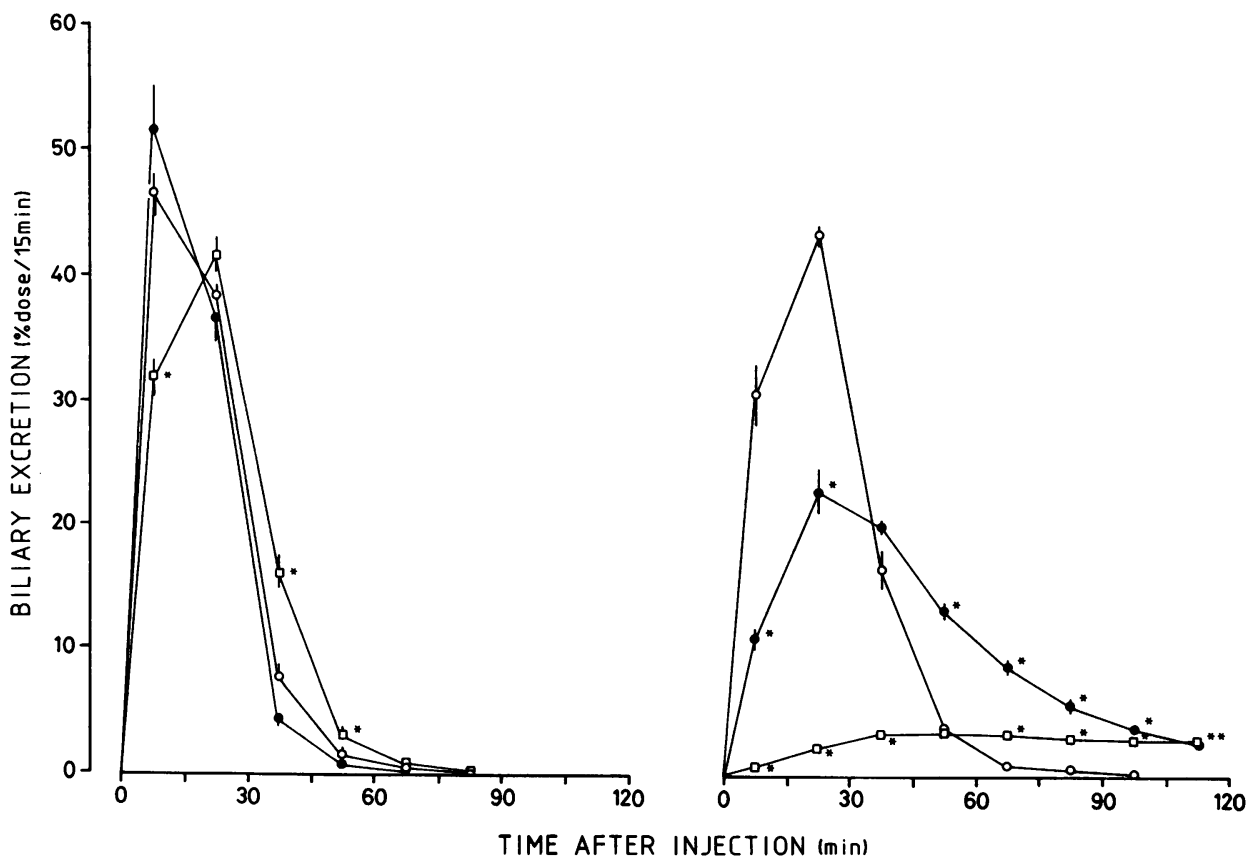

Figure 3. Biliary secretion of ${ }^{3} \mathrm{H}-\mathrm{TC}$ (left) and ${ }^{14} \mathrm{C}$-SGLC (right) after simultaneous administration of trace amounts of both bile acids to jaundiced rats ( $\square$ ), to untreated control rats (o), and to control rats treated with $15 \mu \mathrm{mol} / 100 \mathrm{~g}$ body wt of DBSP, 15 min before administration of the bile acids (๑). Rats were equipped with permanent catheters in bile duct and heart, at least $8 \mathrm{~d}$ before experiments were started. Bile acids were introduced via the heart catheter and bile collection was started in 15-min fractions for 2 h. Values are means $( \pm S E M)$ of four experiments in all groups. 


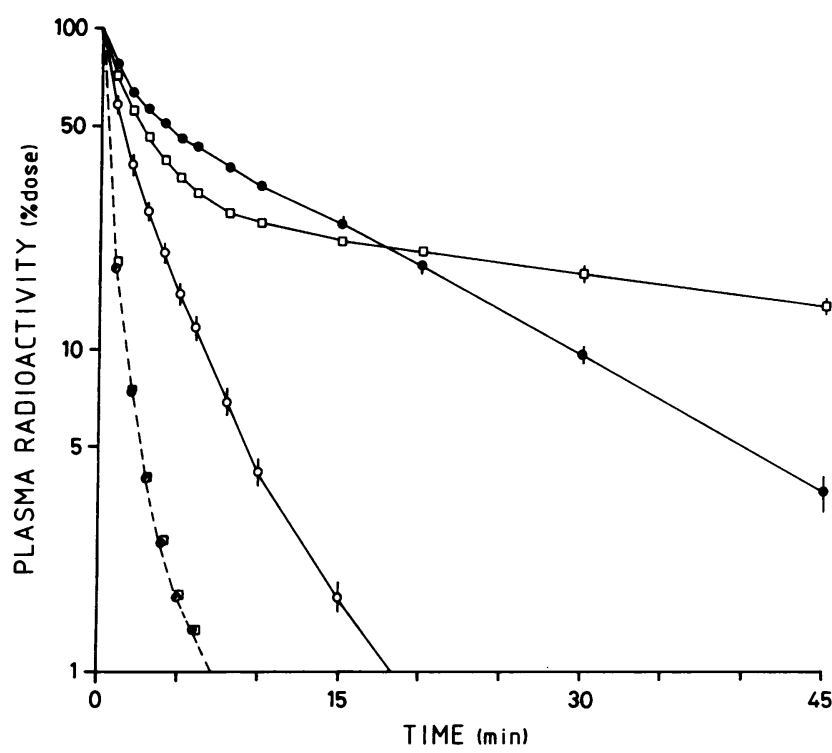

Figure 4. Plasma disappearance of ${ }^{3} \mathrm{H}-\mathrm{TC}$ (dashed line) and ${ }^{14} \mathrm{C}$ SGLC (solid lines), expressed as percentage of dose remaining in plasma, after simultaneous administration of trace amounts of both bile acids to control rats (o), to control rats treated with $15 \mu \mathrm{mol} / 100$ $\mathrm{g}$ body wt of DBSP, 15 min before administration of the bile acids $(\bullet)$, and to jaundiced rats $(\square)$.

acid" organic anion contributes significantly to the BAIF. However, it has been well established that intravenously administered bilirubin does not induce choleresis in rats, not even at maximal bilirubin secretion rates (30). Impaired biliary secretion of other, not yet identified, endogenous compounds may be the cause of the reduction in BAIF.

Table III. Pharmacokinetic Variables of ${ }^{14} \mathrm{C}-S G L C$ Elimination

\begin{tabular}{lccc}
\hline & Jaundiced rats & Control rats & Control rats + DBSP \\
\hline$t^{1 / 2 \alpha}(\mathrm{min})$ & $1.69 \pm 0.06$ & $1.57 \pm 0.15$ & $1.14 \pm 0.14^{*}$ \\
$t / 2 \beta(\mathrm{min})$ & $44.14 \pm 2.88^{*}$ & $4.41 \pm 0.44$ & $11.44 \pm 0.61^{*}$ \\
$V_{1}(\mathrm{ml})$ & $11.96 \pm 0.32$ & $13.38 \pm 0.65$ & $11.09 \pm 0.66$ \\
$V_{2}(\mathrm{ml})$ & $32.62 \pm 1.68^{*}$ & $148.75 \pm 18.23$ & $11.31 \pm 1.31^{*}$ \\
$K_{12}\left(\mathrm{~min}^{-1}\right)$ & $0.297 \pm 0.013$ & $0.390 \pm 0.044$ & $0.287 \pm 0.032$ \\
$K_{21}\left(\mathrm{~min}^{-1}\right)$ & $0.109 \pm 0.004^{*}$ & $0.037 \pm 0.007$ & $0.292 \pm 0.050^{*}$ \\
$K_{20}\left(\mathrm{~min}^{-1}\right)$ & $0.022 \pm 0.002^{*}$ & $0.191 \pm 0.023$ & $0.141 \pm 0.002$ \\
$\mathrm{Cl}(\mathrm{ml} / \mathrm{min})$ & $0.56 \pm 0.03^{*}$ & $4.29 \pm 0.19$ & $1.03 \pm 0.05^{*}$
\end{tabular}

A trace amount of ${ }^{14} \mathrm{C}$-labeled sulfated glycolithocholic $(2 \mu \mathrm{Ci})$ was administered intravenously to jaundiced rats, to untreated control rats, and to control rats that had received $15 \mu \mathrm{mol}$ of DBSP/100 g body wt $15 \mathrm{~min}$ before injection of the bile acid. Radioactivity was determined in plasma samples collected at timed intervals. Data were analyzed by the curve-stripping computer program RUGFIT (25). $t / 2 \alpha$ and $t / 2 \beta$, Elimination half-life for first and second phase; $V_{1}$ and $V_{2}$, distribution volume of central (mainly plasma) and peripheral compartment (mainly liver); $K_{12}$, rate constant for transport from plasma to liver; $K_{21}$, rate constant for transport from liver to plasma $K_{20}$, rate constant for transport from liver to bile; and $\mathrm{Cl}$, total body clearance. Values are means $( \pm$ SEM) from four to five rats in all groups.

* Significantly different $(P<0.05)$ from untreated control rats.
Biochemically and in pattern of inheritance our rats show a great resemblance with the mutant Wistar rats recently described by Jansen et al. (31). Breeding experiments are in progress to investigate whether the two strains carry the same defect.

The overall rate of hepatobiliary transport of cholephilic compounds reflects the rates of their hepatic uptake, intracellular transport, and disposition across the canalicular membrane. Uptake of organic anions has been postulated to occur via at least two independent carrier systems, one for bile acids and another for bilirubin and related organic anions (32). However, in fact overlapping substrate specificity may exist (33). Furthermore, it is not clear whether the transport system for hepatic uptake is common to all bile acids species (34). Carrier-mediated transport systems have also been proposed at the canalicular pole of the hepatocyte $(9,10,33)$.

The presence of a $3 \alpha$-sulfate group introduces an additional negative charge to the bile acid's molecule at physiological pH (35), and alters its polarity and physico-chemical characteristics (36). Sulfation is an important pathway in the metabolism of the secondary bile acid LC. As much as $40-80 \%$ of the relatively small amounts of endogenous GLC and TLC in human bile are present in the form of $3 \alpha$-sulfate esters $(6,37)$. Similar percentages of sulfated LC conjugates were isolated from human bile after oral $(37,38)$ or intravenous $(4)$ administration of radiolabeled LC or GLC. Sulfate esters of di- and trihydroxy bile acids have also been identified in plasma (39, 40 ) and bile (40) of healthy humans, but in relatively much smaller quantities. High concentrations of sulfated bile acids are present in urine of cholestatic patients $(2,3,40)$.

Our results show that, in confirmation with earlier work ( 7 , $8,41), 3 \alpha$-sulfate esters of GLC, TLC, TCDC, and TC are efficiently secreted into bile in normal bile fistula rats. At 60 min after injection of trace amounts of these compounds, $>90 \%$ of the dose was recovered in bile, comparable with the secretion of the corresponding unsulfated compounds. The presence of the sulfate group prevented hepatic metabolism before secretion into bile. The biliary recovery of sulfated bile acids was strongly reduced in the jaundiced rats, the inhibition becoming more pronounced with a decreasing number of hydroxyl groups on the bile acid's molecule. Biliary secretion of unsulfated bile acids was only slightly delayed in the jaundiced rats, which may have been caused by the significantly reduced bile flow in these animals, but the 1-h recovery was similar to that in normal rats. A slightly delayed biliary secretion of endogenous bile acids may be the cause of their elevated plasma concentration in the jaundiced rats (Table II).

Additional experiments were performed to investigate whether impaired uptake, impaired secretion, or a combination of these two factors was responsible for the reduced hepatic transport of sulfates in the jaundiced rats. Uptake and secretion was studied simultaneously with ${ }^{3} \mathrm{H}-\mathrm{TC}$ and ${ }^{14} \mathrm{C}$ SGLC. These compounds were chosen because they are not metabolized before biliary secretion, and they differ maximally in the efficiency of their biliary secretion in the jaundiced rats. Using isolated rat hepatocytes, Bartholomew and Billing (42) demonstrated that uptake of STCDC, TCDC, and sulfated unconjugated CDC occurred by a saturable, energydependent process. Uptake of each of these bile acids was competitively inhibited by the other two. However, TCDC was a very potent inhibitor of uptake of both sulfates, whereas the 
sulfates were relatively poor inhibitors of TCDC uptake. These data suggest the existence of different carriers for sulfated and unsulfated bile acids, with different affinities for both types of compounds. Kinetic data on biliary secretion of bile acid sulfates are scarce. Cleland et al. (7) reported a slightly delayed biliary excretion of STCDC compared with TCDC in bile fistula rats, which contrasts with our findings in long-term bilediverted control rats (Fig. 2), but their results do not exclude differences in initial hepatic uptake between the two compounds. In our experiments, the hepatic handling of TC was not appreciably affected in the jaundiced rats when compared with controls. By contrast, the biliary excretion of SGLC was very drastically reduced. Pharmacokinetic analysis of the plasma disappearance curve revealed that hepatic uptake of SGLC was not affected in the jaundiced animals; its reduced secretion therefore has to be caused by impaired intracellular transport rate and/or defective transport across the canalicular membrane. The rate constant for biliary secretion of ${ }^{14} \mathrm{C}$ SGLC was significantly smaller in the jaundiced rats when compared with controls. Similar, albeit less distinct phenomena were observed in control rats pretreated with DBSP. The dose used saturates the biliary transport of the dye for at least 1 $h$ in the chronic bile fistula rat (17). Interactions between DBSP and bile acids in biliary transport studies have been reported. Bile acids influence hepatic uptake, intracellular binding, and biliary secretion of DBSP; the overall effect depends on the administered amount of bile acids and DBSP (43). We found that DBSP does not interact with hepatic transport of a trace amount of TC in vivo. On the other hand, biliary secretion, but not hepatic uptake, of SGLC was reduced by pretreatment of rats with DBSP. A high rate constant for transport from liver to plasma was found in DBSP-treated rats, due to a very significant reduction of the distribution volume of the second compartment, which was most likely caused by a decreased number of available binding sites for ${ }^{14} \mathrm{C}-\mathrm{SGLC}$.

The presented data show that sulfated bile acids, in particular sulfated LC conjugates, are transported from liver to bile by a system distinct from that for unsulfated bile acids. The sulfates probably share pathways for biliary secretion with organic anions as DBSP and bilirubin. The finding that STC and STCDC are secreted more efficiently than STLC and SGLC in the jaundiced rats (albeit delayed when compared with their unsulfated precursors) may be the result of additional transport of the former compounds by the bile acid transport system. However, other explanations are possible, and further studies on this subject are necessary. Very recently, data have been published on the biliary secretion of the glucuronides of LC (44) and C (45) in the bile fistula rat. Similar to the sulfates, these glucuronides were rapidly secreted into bile. Drop-bydrop collection of bile revealed a slight delay in the biliary secretion of cholate-glucuronide when compared with TC in these experiments, which may also have been caused by involvement of different transport mechanisms.

It has been well established that serum concentrations of unsulfated and sulfated bile acids are elevated in patients with cholestatic liver disease. In a number of clinical studies $(5,46$, 47) it has been observed that the serum concentrations of sulfated bile acids decrease slower after relief of extrahepatic cholestasis than the levels of the unsulfated bile acids. Similar results were obtained by Cleland et al. after relief of bile duct obstruction in the rat (7). In view of our results, these phenom- ena are most likely explained by competition for biliary secretion between the sulfated bile acids and bilirubin conjugates, which will also accumulate in liver and blood during cholestasis. Conversely, competition phenomena may also contribute to the development of hyperbilirubinemia when impaired bile acid secretion leads to the formation of increased amounts of bile acid sulfates (48). The mutant rat provides an excellent model for further investigation of these hepatic transport processes. In addition, it will be useful for studies on hepatic bile formation and on processes involved in the initiation of cholestasis.

\section{Acknowledgments}

The authors thank Sippie Huitema and Albert Gerding for excellent technical assistance, Lucas Vijfschaft for breeding of the "yellow rats," Eddy Ligeon and Jan van der Molen (Laboratory for Clinical Chemistry, University of Groningen) for determination of liver enzymes and serum bile acids, respectively, and Beatrix Gauw for manuscript preparation. Dr. Scaf is thanked for his help in pharmacokinetic analysis.

This work was supported by grant 13-53-44 from Health Organization TNO.

\section{References}

1. Palmer, R. H. 1967. The formation of bile acid sulfates: a new pathway of bile acid metabolism in humans. Proc. Natl. Acad. Sci. USA. 58:1047-1050.

2. Almé, B., A. Bremmelgaard, J. Sjövall, and P. Thomassen. 1977. Analysis of metabolic profiles of bile acids in urine using a lipophilic anion exchanger and computerized gas-liquid chromatography mass spectrometry. J. Lipid Res. 18:339-362.

3. Makino, I., K. Shinozaki, and S. Nakagawa. 1975. Measurement of sulfated and nonsulfated bile acids in urine, serum, and bile of patients with hepatobiliary diseases. Gastroenterology. 68:545-553.

4. Cowen, A. E., M. G. Korman, A. F. Hofmann, and O. W. Cass. 1975. Metabolism of lithocholate in healthy man. I. Biotransformation and biliary excretion of intravenously administered lithocholate, lithocholylglycine, and their sulfates. Gastroenterology. 69:59-66.

5. Dooley, J. S., T. C. Bartholomew, J. A. Summerfield, and B. H. Billing. 1984. The biliary excretion of sulfated and non-sulfated bile acids and bilirubin in patients with external biliary drainage. Clin. Sci. 67:61-68.

6. Stiehl, A., R. Raedsch, G. Rudolph, U. Gundert-Remy, and M. Senn. 1985. Biliary and urinary excretion of sulfated, glucuronidated and tetrahydroxylated bile acids in cirrhotic patients. Hepatology. 5:492-495.

7. Cleland, D. P., T. C. Bartholomew, and B. H. Billing. 1984. Hepatic transport of sulfated and non-sulfated bile acids in the rat following relief of bile duct obstruction. Hepatology. 4:477-483.

8. Kuipers, F., R. Havinga, and R. J. Vonk. 1985. Cholestasis induced by sulphated glycolithocholic acid in the rat: protection by endogenous bile acids. Clin. Sci. 68:127-134.

9. Meier, P. J., A. St. Meier-Abt, C. Barret, and J. L. Boyer. 1984. Mechanisms of taurocholate transport in canalicular and basolateral rat liver plasma membrane vesicles. J. Biol. Chem. 259:10614-10622.

10. Klaassen, C. D., and J. B. Watkins. 1984. Mechanisms of bile formation, hepatic uptake, and biliary excretion. Pharmacol. Rev. 36:1-67.

11. Eyssen, H., J. van Eldere, G. Parmentier, S. Huijghebaert, and J. Mertens. 1985. Influence of microbial bile salt desulfation upon the fecal excretion of bile salts in gnotobiotic rats. J. Steroid Biochem. 22:547-554.

12. Parmentier, G., and H. Eyssen. 1978. Thin-layer chromatography of bile salt sulfates. J. Chromatogr. 152:285-289.

13. Kuipers, F., R. Havinga, H. Bosschieter, G. P. Toorop, F. R. 
Hindriks, and R. J. Vonk. 1985. Enterohepatic circulation in the rat. Gastroenterology. 88:403-411.

14. Hardonk, M. J., F. W. J. Dijkhuis, T. J. Haarsma, J. Koudstaal, and W. A. R. Huijbers. 1977. Application of enzyme histochemical methods to isolated subcellular fractions and to sucrose-Ficoll density gradients. A contribution to the comparison of histochemical and biochemical data. Histochemistry. 54:165-181.

15. Hardonk, M. J., G. Harms, and J. Koudstaal. 1985. Zonal heterogeneity of rat hepatocytes in the in vivo uptake of $17 \mathrm{~nm}$ colloidal gold granules. Histochemistry. 83:473-477.

16. Steffens, A. B. 1969. A method for frequent sampling of blood and continuous infusion of fluids in the rat without disturbing the animal. Physiol. Behav. 4:833-836.

17. Vonk, R. J., E. Scholtens, and J. H. Strubbe. 1978. Biliary excretion of dibromosulphthalein in the freely moving unanaesthetized rat: circadian variations and effects of deprivation of food and pentobarbital anaesthesia. Clin. Sci. Mol. Med. 55:399-406.

18. Wolthers, B. G., M. Volmer, J. C. van der Molen, B. J. Koopman, A. E. J. de Jager, and R. J. Waterreus. 1983. Diagnosis of cerebrotendinous xanthomatosis (CTX) and effect of chenodeoxycholic acid therapy by analysis of urine using capillary gas chromatography. Clin. Chim. Acta. 131:53-65.

19. Blanckaert, N. 1980. Analysis of bilirubin and bilirubin monoand diconjugates. Determination of their relative amounts in biological samples. Biochem. J. 185:115-128.

20. Muraca, M., and N. Blanckaert. 1983. Liquid-chromatography assay and identification of mono- and diester conjugates of bilirubin in normal serum. Clin. Chem. 29:1767-1771.

21. Bligh, E. G., and W. J. Dyer. 1959. A rapid method of total lipid extraction and purification. Can. J. Biochem. Biophys. 37:911-917.

22. Gamble, W., M. Vaughan, M. S. Kruth, and J. Avigan. 1978. Procedure for determination of free and total cholesterol in micro- or nanogram amounts suitable for studies with cultured cells. J. Lipid Res. 19:1068-1071.

23. Bötcher, C. F. J., C. M. van Gent, and C. Pries. 1961. A rapid and sensitive sub micro-phosphorus determination. Anal. Chim. Acta. 24:203-204.

24. Kuipers, F., H. H. Spanjer, R. Havinga, G. L. Scherphof, and R. J. Vonk. 1986. Lipoproteins and liposomes as in vivo cholesterol vehicles in the rat: preferential use of cholesterol carried by small unilamellar liposomes for the formation of muricholic acids. Biochim. Biophys. Acta. 876:559-566.

25. Scaf, A. H. J. 1988. Pharmacokinetic analyses with Rugfit: an interactive pharmacokinetic computer program. Biopharm. Drug Dispos. In press.

26. Kuipers, F., H. Heslinga, R. Havinga, and R. J. Vonk. 1986. Intestinal absorption of lithocholic acid sulfates in the rat: inhibitory effects of calcium. Am. J. Physiol. 251:G189-G194.

27. Schmid, R., J. Axelrod, L. Hammaker, and R. L. Swarm. 1958. Congenital jaundice due to a defect in glucuronide formation. J. Clin. Invest. 37:1123-1130.

28. Dubin, I. N., and F. B. Johnson. 1954. Chronic idiopathic jaundice with unidentified pigment in liver cells. Medicine. 33:155197.

29. Cornelius, C. E., I. M. Arias, and B. I. Osburn. 1965. Hepatic pigmentation with photosensitivity: a syndrome in Corriedale sheep resembling Dubin-Johnson syndrome in man. J. Am. Vet. Med. Assoc. 146:709-713.

30. Van Steenbergen, W., and J. Fevery. 1982. Maximal biliary secretion of bilirubin in the anaesthetized rat: dependence on UDPglucuronosyltransferase activity. Clin. Sci. 62:521-528.
31. Jansen, P. L. M., W. H. Peters, and W. H. Lamers. 1985. Hereditary chronic conjugated hyperbilirubinemia in mutant rats caused by defective hepatic anion transport. Hepatology. 5:573-579.

32. Tiribelli, C., C. C. Lunazzi, and G. L. Sottocasa. 1986. Mechanisms of hepatic uptake of organic anions. Clin. Sci. 71:1-8.

33. Meijer, D. K. F., C. Neef, and G. M. M. Groothuis. 1983. Carrier-mediated transport in the handling of drugs by the liver. In Topics in Pharmaceutical Sciences. D. D. Breimer and P. Speiser, editors. Elsevier Scientific Publishing Co., Amsterdam. 167-189.

34. Anwer, M. S., R. Kroker, and D. Hegner. 1976. Effect of albumin on bile acid uptake by isolated rat hepatocytes. Is there a common bile acid carrier? Biochem. Biophys. Res. Commun. 73:63-71.

35. Donovan, J. M., I. M. Yousef, and M. C. Carey. 1984. Complete sulfation of the common bile salts (BS) of man: synthesis, properties and interactions with lecithin. Gastroenterology. 86:1064. (Abstr.)

36. Carey, M. C., S. F. J. Wu, and J. B. Watkins. 1979. Solution properties of sulfated monohydroxy bile salts. Relative insolubility of the disodium salt of glycolithocholate sulfate. Biochim. Biophys. Acta. 575:16-26.

37. Palmer, R. H., and M. G. Bolt. 1971. Bile acid sulfates. I. Synthesis of lithocholic acid sulfates and their identification in human bile. J. Lipid Res. 12:671-679.

38. Marks, J. W., S. O. Sue, B. J. Pearlman, G. G. Bonorris, P. Varady, J. M. Lachin, and L. J. Schoenfield. 1981. Sulfation of lithocholate as a possible modifier of chenodeoxycholic acid-induced elevations of serum transaminase in patients with gallstones. J. Clin. Invest. 68:1190-1196.

39. Setchell, K. D. R., and A. Matsui. 1983. Serum bile acid analysis. Clin. Chim. Acta. 127:1-17.

40. Stiehl, A., M. Becker, P. Czygan, W. Fröhling, B. Kommerell, H. W. Rotthauwe, and M. Senn. 1980. Bile acids and their sulphated and glucuronidated derivatives in bile, plasma, and urine of children with intrahepatic cholestasis: effects of phenobarbital treatment. Eur. J. Clin. Invest. 10:307-316.

41. Palmer, R. H. 1971. Bile acid sulfates. II. Formation, metabolism, and excretion of lithocholic acid sulfates in the rat. J. Lipid Res. 12:680-687.

42. Bartholomew, T. C., and B. H. Billing. 1983. The effect of 3-sulphation and taurine conjugation on the uptake of chenodeoxycholic acid by rat hepatocytes. Biochim. Biophys. Acta. 754:101-109.

43. Vonk, R. J., M. Danhof, T. Coenraads, A. B. D. van Doorn, K. Keulemans, A. H. J. Scaf, and D. K. F. Meijer. 1979. Influence of bile salts on hepatic transport of dibromosulphthalein. Am. J. Physiol. 237:E524-E534.

44. Oelberg, D. G., M. V. Chari, J. M. Little, E. W. Adcock, and R. Lester. 1984. Lithocholate glucuronide is a cholestatic agent. J. Clin. Invest. 73:1507-1514.

45. Little, J. M., M. V. Chari, and R. Lester. 1985. Excretion of cholate glucuronide. J. Lipid Res. 26:583-592.

46. Bartholomew, T. C., J. A. Summerfield, B. H. Billing, A. M. Lawson, and K. D. R. Setchell. 1982. Bile acid profiles in human serum and skin interstitial fluid and their relationship to pruritus studied by gas chromatography mass-spectrometry. Clin. Sci. 63:65-73.

47. Eklund, A., A. Norlander, and A. Norman. 1980. Bile acid synthesis and excretion following release of total extrahepatic cholestasis by percutaneous transhepatic drainage. Eur. J. Clin. Invest. 10:349-355.

48. Eng, C., and N. B. Javitt. 1983. Chenodeoxycholic acid-3-sulfate. Metabolism and excretion in the rat and hamster and effects on hepatic transport systems. Biochem. Pharmacol. 32:3555-3558. 